\title{
Expression of Vimentin in Spinal Cord Injury
}

\author{
Huang Yumin, Zhou Liang, Lin Weichang* \\ Department of Orthopaedics, the 909th Hospital of PLA, Affiliated Southeast Hospital of Xiamen University, Orthopaedic Center of People's \\ Liberation Army, Zhangzhou, China
}

Email address:

763238730@qq.com (Lin Weichang)

${ }^{*}$ Corresponding author

\section{To cite this article:}

Huang Yumin, Zhou Liang, Lin Weichang. Expression of Vimentin in Spinal Cord Injury. European Journal of Clinical and Biomedical Sciences. Vol. 6, No. 1, 2020, pp. 5-8. doi: 10.11648/j.ejcbs.20200601.12

Received: December 28, 2019; Accepted: January 6, 2020; Published: January 17, 2020

\begin{abstract}
This study is to explore the expression differences of vimentin in the normal spinal cord tissues and the injured spinal cord tissues. The $15 \mathrm{SD}$ rats were randomly divided into two experimental groups and sham group. Acute spinal cord injury models in experimental groups were performed with Lisa method and the injured spinal cord was collected after getting injured 72h. The method of immunohistochemical stain was applied to observe the expressions of vimentin in the normal spinal cord tissue and the injured spinal cord tissue of rats. The results showed the expression of vimentin was found in both the grey matter and white matter of the spinal cord of rats with a relatively even distribution. In addition, the protein expressions of vimentin in the medium injury group and the severe injury group were obviously higher than the sham group. In conclusion, expressions of vimentin were found in the spinal cord, and expression differences were observed after the spinal cord injury, which indicated that vimentin might play a part in the injured process of the spinal cord. It is poised to be a phase of advancement in the treatment of acute spinal cord injury and the importance of further elucidation of underlying pathophysiological mechanisms of vimentin.
\end{abstract}

Keywords: Vimentin, Spinal Cord Injury, Protein Expression

\section{Background}

Besides microfilaments and microtubules, intermediate filaments (IFs) are one of the major structural parts of the cytoskeleton. There has been researched finding out that the cytoskeleton is able to control and regulate multiple cellular functions such as cell contraction, mechanical transmission and genetic expression $[1,2]$. The relative abundance of IFs in cells and the IFs span from the cell nucleus to cytomembrane can both show the essential role of IFs maintaining a stable cell structure and transferring mechanical signals into physiological reactions [3]. In the family of intermediate filaments, vimentin is one of the most well-known members, for it is the main IF protein in the mesenchymal cell and is often used as a marker for the development of cells and tissues. Researches have also discovered that vimentin expressed in a variety of cells, including precursor cells of the pancreas, precursor cells of neurons, trophoblasts macrophages, fibroblasts, endothelial cells, blood vessels, renal tubular cells, macrophages, neutrophilic granulocytes, mechanical cells, leukocytes and renal interstitial cells $[4,5]$. In recent years, studies have found that vimentin can take part in many processes of cells, such as the self-renewal, differentiation, metastasis, adhesion and cell signaling transduction [6]. At the dawning of the present decade, acute spinal cord injury lingers as a formidable disorder affecting society. A fresh surge of studies endeavoring to dissect the cellular and molecular mechanisms involved in the development and perpetuation of acute spinal cord injury appear particularly promising as a basis for identifying candidate pathways of injury for therapeutic manipulation. Meritorious investigations examining changes in gene expression, especially pertaining to pathways of cell death and inflammation, are increasingly reported [7]. But whether vimentin plays any part in the central nervous system damage and repair were not well reported. This study is to detect the expression changes of vimentin protein in the injury process of the spinal cord by making a model of spinal cord injury of rats, so as to explore the possible effects of vimentin on the process of spinal cord injury. 


\section{Materials and Methods}

\subsection{Animal Models and Main Reagents}

A total of 18 female SD rats weighing 220 250g were randomly divided into sham operation (control group) and 2 experimental groups, and 6 rats were in each group. After the intraperitoneal injection of $10 \%$ chloral hydrate $(300 \mathrm{mg} / \mathrm{kg})$ was applied as anesthesia, the T10 segmental spinal cord of the rats should be exposed. Lisa beating method was applied in the experimental group, the depth of spinal cord injury in the moderate injury group was $1.0 \mathrm{~mm}$, while the severe injury group was $1.8 \mathrm{~mm}$. These lower limbs in injured rats were completely paralyzed. After the operation, these rats remained in the room temperature at $20^{\circ} \mathrm{C} \sim 28^{\circ} \mathrm{C}$, and we squeezed the bladder of rats to urinate twice a day without limits of drinking water, feed and illumination. Those in the control group need to be exposed at the T10 segmental spinal cord without injury at spinal cord, while the other treatments were the same as in experimental group. We used the rabbit anti-mouse vimentin antibody.

\subsection{Fixation, Sampling and Section of Tissues}

After getting injured 72 hours, the same anesthesia treating the surgery rats was conducted in the experimental group and in the control group. After applying normal saline to intracardiac perfusion, the injured rats were used $4 \%$ paraformaldehyde for installation. Centering on the injured spinal cord segment, we took a specimen with a length of about $10 \mathrm{~mm}$. The control group was instilled and fixated and took a specimen of the spinal cord with the same length. All the spinal cord tissue needs to be fixed in $4 \%$ paraformaldehyde for 4 hours, put into 30\% sucrose PBS solution, and cut frozen sections with a thickness of $10 \mu \mathrm{m}$ after the tissue sediment.

\subsection{Immunologic Tissue Fluorescence}

After spinal cords were injured 72 hours, we took the injured spinal cord tissues for the immunohistochemical staining. Then we put the sampled spinal cord tissues into $20 \%$ and $30 \%$ sucrose solution in order for the gradient of dehydration and make them into frozen sections with a thickness of $14 \mu \mathrm{m}$. The injured spinal cord tissues have put the sections into $3 \%$ Methanol $-\mathrm{H}_{2} \mathrm{O}_{2}$ solution and incubated for 30 minutes at a temperature of $37^{\circ} \mathrm{C}$, then washed with $\mathrm{PBS}$ for three times and put into antiserum blocking fluid and incubate for another 2 hours at indoor temperature. And these tissues were resorbed in the redundant blocking fluid, add the primary antibodies and incubated for another 1 hour at normal temperature, then we put them at a temperature of $4^{\circ} \mathrm{C}$ during whole night. Then it was incubated the second antibody for 1 hour at a temperature of $37^{\circ} \mathrm{C}$. Finally, we applied hematoxylin to counterstaining, dehydration, clearing and mounting. Use PBS to replace the primary antibody as the negative control, and the light microscope should be implemented to count the positive cells.

\section{Results}

Immunofluorescence staining of spinal cord tissue

According to the results, vimentin had expressions in both grey matter and white matter of normal spinal cord with a relatively even distribution. In 72 hours after the injury, the positive cell expressions of Vimentin in both grey matter and white matter obviously increased and the positive cells were dramatically more than the control group (Figure 1).
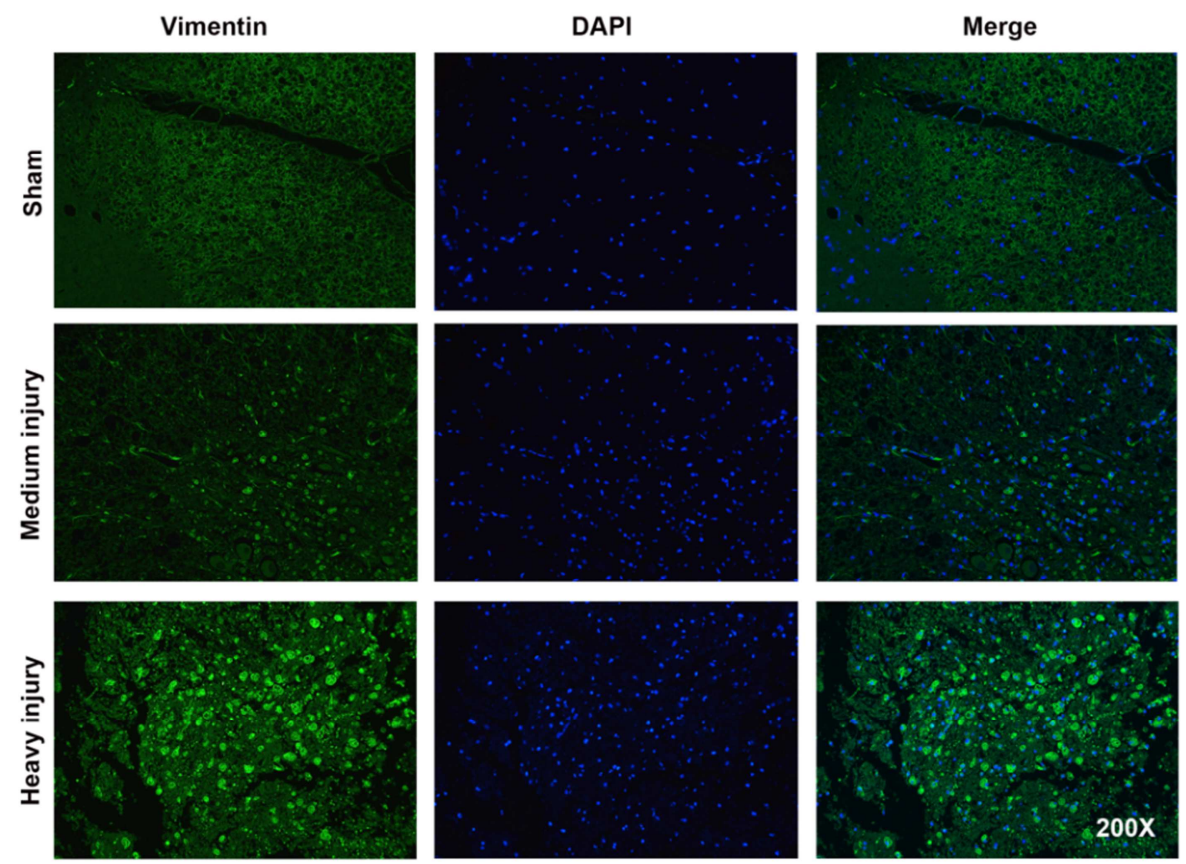

Figure 1. Immunofluorescence staining of spinal cord tissue. Anti-vimentin were used to detect endogenous proteins in the normal spinal cord of control group (Sham) and injured spinal cord of the medium injury group and heavy injury group. Dapi is utilized to show the location of the nucleus. Merge pictures represent the distribution of vimentin in cells. The magnification of the picture is 200 times. 


\section{Discussion}

After acute spinal cord injury, the myelin sheath and axon in the damage zone were disintegrated and broken, and there was active proliferation of gliocyte and scar. After that, the axon near the segment of injury sprouted and extended. During the process including regeneration and repair of nerve fiber, the movement of the cytoskeleton was crucial [8]. Intermediate filament protein is one of the three major cytoskeleton proteins in eukaryotes, including keratin, desmin, vimentin, glial fibrillary acidic and neurofilament protein [9]. In this study, the focus is paid to one of the intermediate filament proteins with the wildest expressions of vimentin.

As a class-III intermediate filament, vimentin is characterized by its high content and distinct conservative property. The complex structure and diversified functions of vimentin are supported by the modifying of phosphorylation, which at the same time is able to help vimentin receive and transmit signals [10]. The dimer formed by spiral winding in the central rod-like domain is an important part of the intermediate filaments of vimentin. Two dimer structures are wound parallel to each other to form a new tetramer structure, with more tetramers forming thin, long intermediate filaments, such a structure is the basic structure of a cytoskeleton [11]. Both outside and inside of an organism, the individual of vimentin can automatically compound the vimentin fiber in the first place. The vimentin fiber manages to balance between polymerization and depolymerization via the process of phosphorylation and dephosphorylation, and such a stability is achieved only when class-III IFs cooperates with protein Clathrin. Any changes of vimentin such as recombination and disintegration may cause changes of physiological function or even pathological changes to an organism. The tissues of filiform cells of vimentin are different in different cells. Vimentin has been proved to be an important element in diverse biological processes such as cell survival, apoptosis-inducing, restraining differentiation, promoting cell invasion and metastasis $[6,12,13]$.

According to the results of this experiment, there was an even distribution of vimentin in the grey matter and white matter of spinal cord. After the injury of spinal cord, the expressions of vimentin had been changing along with the degree of injury. There were temporal and spatial variation features in the expressions of vimentin, which indicated that vimentin might play a part in the injury and recovery of spinal cord. The division of vimentin would cause irreversible damage to the intermediate filament, accelerating the cell apoptosis, and at this stage, much assistance of signal path would be needed. Rho A/ROCK and raf1 /CK2 path have participated in numerous cell functions, including cell proliferation, apoptosis, cell differentiation and so on. Under a vimentin cytoskeleton, the Raf-1-CK2 and Rho A-Rho Kinase signal can accelerate the cell apoptosis with the assistance of tumor necrosis factor $\alpha$ [14]. Vimentin can be hydrolyzed into vimentin amino-terminal fragment to accelerate apoptosis, which can induce cell apoptosis, vimentin degradation or induced to generate caspase apoptosis signal, accelerating the cell apoptosis as well. After being activated, the caspase family would decompose vimentin into fragments and particulate matters, which were distributed around the cell nucleus. The fragments generated would activate caspase, leading to cascade amplification of the apoptosis signals $[15,16]$, which indicated the effects on regulating the apoptosis and proliferation of corresponding cells. However, the specific mechanism that vimentin applies to participate in the injury and recovery of the spinal cord is still what we should study and research in the future.

\section{Conclusion}

In conclusion, expressions of vimentin were found in both the grey matter and white matter of the spinal cord, and expression changes were observed after the spinal cord injury, which indicated that vimentin might play a part in the injured process of the spinal cord. It is poised to be a phase of advancement in the treatment of acute spinal cord injury and the importance of further elucidation of underlying pathophysiological mechanisms of vimentin.

\section{References}

[1] Chen J, Fabry B, Schiffrin EL, et al. Twisting integrin receptors increases endothelin-1 gene expression in endothelial cells. American journal of physiology Cell physiology 2001, 280 (6): C1475-1484.

[2] Moujaber O, Stochaj U. The Cytoskeleton as Regulator of Cell Signaling Pathways. Trends in biochemical sciences 2019.

[3] Duncan EJ, Lariviere R, Bradshaw TY, et al. Altered organization of the intermediate filament cytoskeleton and relocalization of proteostasis modulators in cells lacking the ataxia protein sacsin. Human molecular genetics 2017, 26 (16): 3130-3143.

[4] De Souza PC, Katz SG. Coexpression of cytokeratin and vimentin in mice trophoblastic giant cells. Tissue \& cell 2001, 33 (1): 40-45.

[5] Ko SH, Suh SH, Kim BJ, et al. Expression of the intermediate filament vimentin in proliferating duct cells as a marker of pancreatic precursor cells. Pancreas 2004, 28 (2): 121-128.

[6] Benes P, Maceckova V, Zdrahal Z, et al. Role of vimentin in regulation of monocyte/macrophage differentiation. Differentiation; research in biological diversity 2006, 74 (6): 265-276.

[7] Dumont AS, Dumont RJ, Oskouian RJ. Will improved understanding of the pathophysiological mechanisms involved in acute spinal cord injury improve the potential for therapeutic intervention? Current opinion in neurology 2002, 15 (6): 713-720.

[8] Werner HB, Nave KA. Enhanced Actin Dynamics: A Therapeutic Strategy for Axonal Regeneration? Neuron 2019, 103 (6): 949-950. 
[9] Hol EM, Capetanaki Y. Type III Intermediate Filaments Desmin, Glial Fibrillary Acidic Protein (GFAP), Vimentin, and Peripherin. Cold Spring Harbor perspectives in biology 2017, 9 (12).

[10] Parry DA. Hendecad repeat in segment 2A and linker L2 of intermediate filament chains implies the possibility of a right-handed coiled-coil structure. Journal of structural biology 2006, 155 (2): 370-374.

[11] Dey R, Burkhard P. A proposed atomic model of the head-to-tail interaction in the filament structure of vimentin. Journal of biomolecular structure \& dynamics 2019: 1-7.

[12] Deng Z, Du WW, Fang L, et al. The intermediate filament vimentin mediates microRNA miR-378 function in cellular self-renewal by regulating the expression of the Sox2 transcription factor. The Journal of biological chemistry 2013, 288 (1): 319-331.
[13] Lin J, Lu J, Wang C, et al. The prognostic values of the expression of Vimentin, TP53, and Podoplanin in patients with cervical cancer. Cancer cell international 2017, 17: 80.

[14] Yang L, Tang L, Dai F, et al. Raf-1/CK2 and RhoA/ROCK signaling promote TNF-alpha-mediated endothelial apoptosis via regulating vimentin cytoskeleton. Toxicology 2017, 389: 74-84.

[15] Byun Y, Chen F, Chang R, et al. Caspase cleavage of vimentin disrupts intermediate filaments and promotes apoptosis. Cell death and differentiation 2001, 8 (5): 443-450.

[16] Su L, Pan P, Yan P, et al. Role of vimentin in modulating immune cell apoptosis and inflammatory responses in sepsis. Scientific reports 2019, 9 (1): 5747. 